\title{
New Insights from a Bounded Rationality Analysis for Strategic Price-QoS War
}

\author{
Mohamed Baslam*, Rachid El-Azouzi ${ }^{\dagger}$, Essaid Sabir ${ }^{\ddagger}$ and El-Houssine Bouyakhf ${ }^{\S}$ \\ *LIP (UMR CNRS - ENS de Lyon - UCB Lyon 1 - INRIA 5668), Université de Lyon, France. \\ Email: mohamed.baslam@ens-lyon.fr \\ ${ }^{\dagger}$ LIA-CERI, University of Avignon, 339 chemin des Meinajaries, Agroparc BP 1228, 84911 Avignon cedex 9, France. \\ Email: rachid.elazouzi@univ-avignon.fr

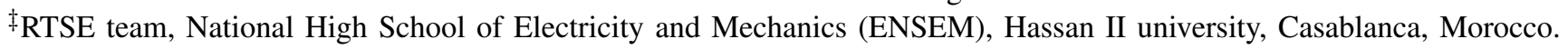 \\ Email: e.sabir@ensem.ac.ma \\ $\S$ LIMIARF, Faculty of Sciences, University of Mohammed V-Agdal, 4 Avenue Ibn Battouta B.P. 1014 RP, Rabat, Morocco. \\ Email: bouyakhf@mtds.com
}

\begin{abstract}
We present a game theoretic framework for the dynamical behaviors of a duopoly game in telecommunications service providers' context. Competition between two Service Providers (SPs) is assumed to take place in terms of their pricing decisions and the Quality of Service (QoS) they offer. According to the SPs' rationality level, we consider two schemes: 1) Both SPs are rational, and 2) One SP is rational and the second SP is boundedly rational. We describe the competitive interaction and analyze the resulting equilibria. Later, we compute explicitly the steady states of the dynamical system induced by bounded rationality, and establish a necessary and sufficient condition for stability of its Nash equilibria (NEs). We prove that there exists exactly one $\mathrm{NE}$ which is fair whereas remaining equilibria are unfair. A special feature is that the stability condition of the Nash equilibrium coincides with the instability condition of the boundary equilibria. Thus the system would never be absorbed by any of the unfair equilibria which solves the equilibrium selection issue ! Moreover, we show that considering the delay case (i.e., assuming a market with memory) increases the stability of the system. Here, the size of the memory could be considered for multi-level rationality, which means that bounded rationality tends to rationality as the memory size increases. We finally show that boundedly rational SPs with delay have a higher chance of reaching the fair Nash equilibrium.
\end{abstract}

Index Terms-Pricing, QoS, Nash equilibrium, bounded rationality, delay, Stability.

\section{INTRODUCTION}

One of the most fundamental concepts and/or assumptions in game theoretic models is rationality. Rationality implies that every player is reasoned by increasing his own payoff, in other word, all information concerning the game is known to all players, therefore, everyone is acting rationally, and so, they are capable to maximize their own payoff. John V. Neumann and Morgenstern justified the idea of maximizing the expected payoff in their work [3]. In the real world, the assumption of "full rationality" almost never holds. This realworld "bounded rationality" is one of the major impediments to applying conventional game theory in the real world. To make those predictions, it is necessary to first have some information/data concerning the system, to serve as the basis of one's prediction. Without such an information, science could say nothing, and to pretend otherwise is erroneous. In the real-world, bounded rationality strategy means that the player makes its output decision from some partial information. In the economic literature, and as pointed out by several authors, [5]-[7], [9], partial information could efficiently be modeled as a marginal profiles. Namely, players decide their strategy according to their respective marginal profit.

The competitive model in economics or/and telecommunications related issues assume generally a single parameter game, [1], [5]-[7]. Yet, it is important and more realistic to incorporate into the model more than one strategic parameter. In this paper, we consider a telecommunication market shared by two SPs. On one hand, each SP charges a tariff (price) for the services it offer. On the other hand, each SP guarantees some given QoS. The QoS metric could be delay, throughput, loss probability, etc.. Later, we build a mathematical framework where we include both price and some measure of the perceived QoS. Although the problem becomes more complicated, it provides many insightful results for the market share game in telecommunication context. Next,we study the general problem of joint price and QoS game with bounded rationality. Each SP (player) acts under bounded rationality and adjusts his price and QoS strategic parameters according to its expected marginal profit. The study of this problem enables us to realize that there exists more than one fixed point of the nonlinear system. In some of them, one SP gets zero payoff, we refer to those equilibria as boundary points. Boundary points are definitely unfair, then the market owner would act so as to eliminate them by fulfilling their instability conditions. We proved that at most one fair fixed point exists, we will refer to this point as the Nash equilibrium of the duopoly game. This latter is characterized by a non-zero output of both SPs. The stability of Nash equilibrium is also studied. A special feature is that the stability condition of the Nash equilibrium coincides with the instability condition of the boundary equilibria. Thus the system would never be absorbed by any of the unfair equilibria which solves the equilibrium selection problem ! In other words, the boundary equilibria are unstable. Moreover, we introduce the delayed bounded rationality to enable us to 
show that the memory size of the market share game increases the stability of Nash equilibrium.

This paper is organized as follows: In Section II, we provide a detailed description of duopoly game with bounded rationality model. In Section III, we have presented a dynamic model with bounded rationality using one parameter price game, existence and stability of Nash equilibrium and the boundary equilibria are studied. In Section IV, we have studied the duopoly game with bounded rationality in presence of price and QoS parameters. Finally, conclusions and future guidelines are drawn in Section V.

\section{PROBLEM FORMULATION}

We formulate the interaction between two SPs as a noncooperative game. Each SP chooses the QoS to guarantee (depends on the amount of requested bandwidth) and the corresponding price.

In this paper we consider a duopoly game which describes a market with two SPs, who decide their price and QoS strategies in order to maximize their incomes. Let $p_{i}$ and $q_{i}$ be, respectively, the tariff/pricing policy and the QoS guaranteed by $\mathrm{SP} i$. Now, each customer seeks to subscribe to the operator which allows him to meet a QoS sufficient to satisfy his needs, at adequate price. We consider that customers' behaviors has been handled by a simple function so called demand functions, see equation (1). This later depends on the price and QoS strategies of all SPs. From a tagged SP's point of view, the question is to set the best pricing strategy and the best QoS (amount of bandwidth to request from the network owner). SPs are supposed to know the effect of their policy on the customers subscription policy. Whereas from customers point of view, the question is to find the SP that has the best priceQoS tradeoff conditions.

\section{A. Demand model}

We consider that the demand function $D_{i}$ for services of $\mathrm{SP} i$ is linear with respect to the set price $p_{i}$ and the promised QoS $q_{i}$, see [2]. This demand function depends also on prices $\mathbf{p}_{-i}$ and QoS $\mathbf{q}_{-i}$ set by the competitors. Namely, the demand function of SP $i$ depends on $\mathbf{p}=\left[p_{1}, p_{2}\right]$ and $\mathbf{q}=\left[q_{1}, q_{2}\right]$. Eventually, $D_{i}$ is decreasing w.r.t. $p_{i}$ and increasing w.r.t. $p_{j}, \quad j \neq i$. Whereas it is increasing w.r.t $q_{i}$ and decreasing w.r.t. $q_{j}, j \neq i$. Then, the demand functions w.r.t services of SP $i$ can be written as follows:

$$
D_{i}(\mathbf{p}, \mathbf{q})=D_{i}^{0}-\alpha_{i}^{i} p_{i}+\beta_{i}^{i} q_{i}+\alpha_{i}^{j} p_{j}-\beta_{i}^{j} q_{j}, \quad j \neq i .
$$

where $D_{i}^{0}$ is a positive constant used to insure non-negative demands over the feasible region. While $\alpha_{i}^{j}$ and $\beta_{i}^{j}$ are positive constants representing respectively the sensitivity of $\mathrm{SP} i$ to price and QoS of service provider $j$.

We consider the following assumptions, which will be useful throughout this work :

Assumption 1: For any pricing profile, the price mutual sensitivities satisfy:

$$
\alpha_{i}^{i} \geq \alpha_{i}^{j}, \quad \forall i \neq j \in\{1,2\} .
$$

Assumption 1 is a reasonable condition, because it implies that the influence of an SP's price is significantly greater on its observed demand than the prices of its competitor.

Assumption 2: We assume that the demand function of each SP-i satisfies, for all $i \in\{1,2\}$,

$$
D_{i}\left(p^{\max }-p_{i}^{\min }, 0_{-i}, \mathbf{q}\right) \leq 0, \quad \forall \mathbf{q},
$$

where $p_{i}^{\text {min }}$ represents the minimum price in the strategy space of SP-i and $p^{\max }$ is the maximum price in the strategy space of both SPs.

In view of demand function in equation (1), and according to Assumption 1, if we choose $p^{\max }$ large enough then Assumption 2 will hold.

\section{B. Utility model}

The net revenue of SP- $i$ is the difference between its total income, $p_{i} D_{i}(\mathbf{p}, \mathbf{q})$, minus its total outcome. We assume that we have a single network owner, this latter charges each SP- $i$ a cost $\vartheta_{i}$ per unit of requested bandwidth. In order to insure the customers loyalty, the amount of bandwidth $\mu_{i}$ purchased by SP- $i$ should depend on $D_{i}($.$) and on the QoS q_{i}$ it wishes to guarantee to its customers, so

$$
\Pi_{i}(\mathbf{p}, \mathbf{q})=D_{i}(\mathbf{p}, \mathbf{q}) p_{i}-F_{i}\left(q_{i}, D_{i}\right), \quad \forall i \in\{1,2\} .
$$

where $F_{i}\left(q_{i}, D_{i}\right)$ is a total fee paid by SP to get bandwidth $\mu_{i}: F_{i}=\vartheta_{i} \mu_{i}\left(q_{i}, D_{i}\right)$.

As already assumed in [2], we consider that the amount of bandwidth required by user SP- $i$ has the following form:

$$
\mu_{i}\left(q_{i}, D_{i}\right)=D_{i} g_{i}\left(q_{i}\right)+h_{i}\left(q_{i}\right) .
$$

where $g_{i}($.$) and h_{i}($.$) are positive functions.$

So, the utility function of SP- $i$ has the following form:

$\Pi_{i}(\mathbf{p}, \mathbf{q})=D_{i}(\mathbf{p}, \mathbf{q})\left(p_{i}-\vartheta_{i} g_{i}\left(q_{i}\right)\right)-\vartheta_{i} h_{i}\left(q_{i}\right), \quad \forall i \in\{1,2\}$.

Later, and in order to have a closed formula of the utility function, we will restrict to some special cases where QoS is simple enough (e.g. QoS as expected delay or QoS as Loss or rejection probability).

\section{Rationality and bounded rationality}

In game theory, players are rational if each one knows all information and rules which allow to maximize his incomes and minimize his costs, or certainly choose their best response, and so, he is able to deduce all the consequences of his decision. Most of the game theorists assume that players are rational. A common example is the pricing issue in economics field. In the general equilibrium model of price theory, [1], [2], it is assumed that every player is rational, but it is not assumed that players understand the whole structure of the economic model that the price theorist is studying.

It is clear that the mechanism of rationality does not represent perfectly the interactions (actions and reactions) of players in the market. A more realistic approach is to assume a bounded rationality mechanism, the latter assumes that players do not have sufficient capacities and information 
to choose rational strategies in the sense of maximizing their utilities. Instead, the players choose an action by means of some heuristic rules. This sort of choosing is called "bounded rationality".

In this paper, we assume that players are boundedly rational, and their rationality is limited by the information that they have. We focus on a player's ability to anticipate the reaction of its opponent, so, the bounded rationality is defined as follow: when a player at given time, $t$, decide his price/QoS strategies, he does not know how the competitor reacts at time $t+1$, and so, he does not know the benefit that it may have, because this latter depends on the reaction of the other player. However, as pointed out by Bischi and Naimzada [9], the bounded rationality strategy means that the player makes its output decision in terms of the local estimate of the marginal profit. Namely, one player decides to increase its strategy (Price or QoS) if it has a positive marginal profit, or decreases its strategy if its marginal profit is negative. Hence, if we consider single parameter price game, the dynamics of the duopoly price game has the following forme:

$$
\left\{p_{i}(t+1)=p_{i}(t)+\theta_{i} p_{i}(t) \frac{\partial \Pi_{i}(t)}{\partial p_{i}}, \quad i \in\{1,2\}\right.
$$

where $\theta_{i}, \quad i=1,2$ is a positive parameter which represents the speed of adjustment.

To find out the impact of rationality of players on the competition of market, we will study two different scenarios:

1) Asymmetric Scenario: That arise when an SP is rational (he knows all the market information), while the other is boundedly rational.

2) Symmetric Scenario: When both SPs are Boundedly rational.

The third possible scenario is the case when both SPs are rational, this case is already studied in [1] and [2].

\section{PRICE-BASED GAME}

In this section, we assume that the vector of QoS parameters, $\mathbf{q}$, of all SPs is fixed at some predetermined point, $\overline{\mathbf{q}}$, and we consider only the price game. At the discrete time, $t$, the profit of SP- $i$ is given by:

$$
\Pi_{i}(t)=D_{i}(t)\left(p_{i}(t)-\vartheta_{i} \bar{g}_{i}\right)-\vartheta_{i} \bar{h}_{i}, \quad \forall i \in\{1,2\},
$$

where $\bar{g}_{i}=g_{i}\left(\bar{q}_{i}\right)$ and $\bar{h}_{i}=h_{i}\left(\bar{q}_{i}\right)$ are positive real constants. In the following, we would like to analyze the impact of rationality on the competition in the market, so we shall proceed as follows: First, we examine the asymmetric scenario, in this latter, we consider that SP-1 is rational, whereas SP-2 is boundedly rational. After that, we will study the symmetric scenario, when both SPs are boundedly rational.

\section{A. Asymmetric Scenario}

In this scenario, we distinguish between two types of players. On the one hand, we assume that SP-1 is rational, it means that he has knowledge of how his competitor will react to his decision, the second derivative of the utility function of $\mathrm{SP}-1$ is given by

$$
\frac{\partial^{2} \Pi_{1}(\mathbf{p}, \overline{\mathbf{q}})}{\partial p_{1}^{2}}=-2 \alpha_{1}^{1}<0,
$$

this mean that the utility function of SP-1 is concave. Thereafter, we shall show that at strategy price that maximizes profit of SP-1 $\left(p_{1}^{*}\right)$, the optimality condition : $\frac{\partial \Pi_{1}(\mathbf{p}, \overline{\mathbf{q}})}{\partial p_{1}}=0$, is checked. To do, it suffices to prove that the point $p_{1}^{*}$ is an internal point of the interval $] p_{1}^{\min }, p^{\max }\left[\right.$, where $p^{\max }$ is the maximum price, and $p_{1}^{\min }$ is minimum price of SP1. We note that, $p_{1}^{\min }$, is a function of the QoS fixed by SP-1, $\bar{q}_{1}$, this condition will allow us to eliminate explicitly strategies that will result in negative profits for SP-1, so, in view of profits formula in equation (5) we deduce that : $p_{1}^{\min }=\vartheta_{1} g_{1}\left(\bar{q}_{i}\right)=\vartheta_{1} \bar{g}_{1}$. Besides, we have

$$
\begin{aligned}
\lim _{p_{1} \rightarrow \vartheta_{1} \bar{g}_{1}} \frac{\partial \Pi_{1}\left(p_{1}, p_{2}, \overline{\mathbf{q}}\right)}{\partial p_{1}}= & D_{1}\left(\vartheta_{1} \bar{g}_{1}, p_{2}, \overline{\mathbf{q}}\right) \geq 0 \\
\lim _{p_{1} \rightarrow p^{\max }} \frac{\partial \Pi_{1}\left(p_{1}, p_{2}, \overline{\mathbf{q}}\right)}{\partial p_{1}}= & -\alpha_{1}^{1} p^{\max }+\alpha_{1}^{1} \vartheta_{1} \bar{g}_{1}+D_{1}\left(p^{\max }, p_{2}, \overline{\mathbf{q}}\right) \\
= & D_{i}^{0}-2 \alpha_{1}^{1} p^{\max }+\beta_{1}^{1} \bar{q}_{1}+\alpha_{1}^{2} p_{2}-\beta_{1}^{2} \bar{q}_{2} \\
& +\alpha_{1}^{1} \vartheta_{1} \bar{g}_{1} \\
\leq & D_{i}^{0}-2 \alpha_{1}^{1} p^{\max }+\alpha_{1}^{2} p^{\max }+\beta_{1}^{1} \bar{q}_{1}-\beta_{1}^{2} \bar{q}_{2} \\
& +\alpha_{1}^{1} \vartheta_{1} \bar{g}_{1} \\
= & D_{i}^{0}-\alpha_{1}^{1} p^{\max }+\left(-\alpha_{1}^{1}+\alpha_{1}^{2}\right) p^{\max }+\beta_{1}^{1} \bar{q}_{1} \\
& -\beta_{1}^{2} \bar{q}_{2}+\alpha_{1}^{1} \vartheta_{1} \bar{g}_{1} \\
< & D_{i}^{0}-\alpha_{1}^{1} p^{\max }+\beta_{1}^{1} \bar{q}_{1}-\beta_{1}^{2} \bar{q}_{2} \\
& +\alpha_{1}^{1} \vartheta_{1} \bar{g}_{1} \\
= & D_{1}\left(p^{\max }-\vartheta_{1} \bar{g}_{1}, 0, \overline{\mathbf{q}}\right) \\
< & 0
\end{aligned}
$$

the transition from (8) to (9) is based on the Assumption 1 whereas the last transition is due to Assumption 2. From (7) and (10) we have that: $\left.p_{1}^{*} \in\right] p_{1}^{\min }, p^{\max }\left[\right.$, thus, $p_{1}^{*}$ is a unique solution of the optimality condition:

$$
\frac{\partial \Pi_{1}(\mathbf{p}, \overline{\mathbf{q}})}{\partial p_{1}}=0
$$

so, to maximize its profit, SP-1, will choose his best response strategy price according to the first order conditions:

$$
p_{1}=\frac{C_{1}+\alpha_{1}^{2} p_{2}}{2 \alpha_{1}^{1}},
$$

where $C_{1}=D_{0}^{1}+\alpha_{1}^{1} \vartheta_{1} \bar{g}_{1}+\beta_{1}^{1} \bar{q}_{1}-\beta_{1}^{2} \bar{q}_{2}$.

On the other hand we consider that SP-2 is boundedly rational, and as mentioned by Bischi and Naimzada [9], a bounded rational player modifies its output decisions according to its marginal profit: $\frac{\partial \Pi_{2}(\mathbf{p})}{\partial p_{2}}$. The dynamical equation for decisions of SP-2 has the form:

$$
p_{2}(t+1)=p_{2}(t)+\theta_{2} p_{2}(t) \frac{\partial \Pi_{2}\left(p_{1}^{e}(t+1), p_{2}(t)\right)}{\partial p_{2}}(12)
$$

such that $p_{1}^{e}(t+1)$ is expected price of SP-1 in the next time step $(t+1)$. Because of the dynamic, the price decided by the SP-2 in equation (12) may exceed the maximum price $p^{\max }$, or it may be below the minimum price $p_{2}^{\min }$, to prevent this excess, we project equation (12) on the interval $\left[p_{2}^{\min }, p^{\max }\right]$, so, equation (12) becomes:

$p_{2}(t+1)=\left[p_{2}(t)+\theta_{2} p_{2}(t) \frac{\partial \Pi_{2}\left(p_{1}^{e}(t+1), p_{2}(t)\right)}{\partial p_{2}}\right]_{p_{2}^{\min }}^{p^{\max }}$ 
From (11) and (13), the dynamical system of a duopoly game with asymmetric scenario is:

$$
\left\{\begin{aligned}
p_{1}(t+1)= & \frac{C_{1}+\alpha_{1}^{2} p_{2}(t+1)}{2 \alpha_{1}^{1}} \\
p_{2}(t+1)= & p_{2}(t)+\theta_{2} p_{2}(t)\left[-\alpha_{2}^{2}\left(p_{2}(t)-\vartheta_{2} \bar{g}_{2}\right)\right. \\
& \left.+D_{2}\left(p_{1}^{e}(t+1), p_{2}(t)\right)\right] .
\end{aligned}\right.
$$

As it was considered by Gibbons in [10], it makes sense to assume that in the bounded rationality term, the expected price $p_{i}^{e}(t+1)$ decided by $\mathrm{SP}-i$ is equal to its previous value $p_{i}(t)$. However to anticipate $p_{i}^{e}(t+1)$, it may make more sense to assume that SPs each one has a memory for storing prices decided in previous slot time before time, $t$. So to anticipate the expected price of his competitor at $(\mathrm{t}+1)$, SP-2 use previous prices i.e. $p_{1}(t-1), p_{1}(t-2), \ldots, p_{1}(t-T)$ with different weights, this point of view has been studied [6] [11] in different contexts. Generally, the expected price of SP- $i$ become: $p_{i}^{e}(t+1)=\sum_{l=0}^{T} \omega_{l} p_{i}(t-l), \quad \omega_{l} \geq 0 \quad$ and $\quad \sum_{l=0}^{T} \omega_{l}=1$, the constants $\omega_{l}, \quad l=0,1, \ldots, T$ are the weights given to previous prices and $T$ represents the size of memory, so (14) become:

$$
\left\{\begin{aligned}
p_{1}(t+1)= & \frac{C_{1}+\alpha_{1}^{2} p_{2}(t+1)}{2 \alpha_{1}^{1}} \\
p_{2}(t+1)= & p_{2}(t)+\theta_{2} p_{2}(t)\left[-2 \alpha_{2}^{2} p_{2}(t)+C_{2}\right. \\
& \left.+\alpha_{2}^{1}\left(\sum_{l=0}^{T} \omega_{l} p_{1}(t-l)\right)\right]
\end{aligned}\right.
$$

where $C_{2}=D_{0}^{2}+\alpha_{2}^{2} \vartheta_{2} \bar{g}_{2}+\beta_{2}^{2} \bar{q}_{2}-\beta_{2}^{1} \bar{q}_{1}$.

For simplicity we set $(T=1)$, in this case the previous dynamical model, with one step $(T=1)$ is given by:

$$
\left\{\begin{aligned}
p_{1}(t+1)= & \frac{C_{1}+\alpha_{1}^{2} p_{2}(t+1)}{2 \alpha_{1}^{1}} \\
p_{2}(t+1)= & p_{2}(t)+\theta_{2} p_{2}(t)\left[-2 \alpha_{2}^{2} p_{2}(t)+C_{2}\right. \\
& \left.+\alpha_{2}^{1}\left(\omega_{1} p_{1}(t)+\left(1-\omega_{1}\right) p_{1}(t-1)\right)\right] .
\end{aligned}\right.
$$

To study the stability of (15), we rewrite it as a third dimensional system in the form

$$
\left\{\begin{aligned}
r_{1}(t+1)= & p_{1}(t) \\
p_{1}(t+1)= & \frac{C_{1}+\alpha_{1}^{2} p_{2}(t+1)}{2 \alpha_{1}^{1}} \\
p_{2}(t+1)= & p_{2}(t)+\theta_{2} p_{2}(t)\left[-2 \alpha_{2}^{2} p_{2}(t)+C_{2}\right. \\
& \left.+\alpha_{2}^{1}\left(\omega_{1} p_{1}(t)+\left(1-\omega_{1}\right) r_{1}(t)\right)\right] .
\end{aligned}\right.
$$

The dynamic system (16) has two fixed points in the following form:

$$
\begin{aligned}
& E_{1}=\left(\frac{C_{1}}{2 \alpha_{1}^{1}}, \frac{C_{1}}{2 \alpha_{1}^{1}}, 0\right) \\
& E_{*}=\left(\frac{2 \alpha_{2}^{2} C_{1}+\alpha_{1}^{2} C_{2}}{4 \alpha_{2}^{2} \alpha_{1}^{1}+\alpha_{2}^{1} \alpha_{1}^{2}}, \frac{2 \alpha_{2}^{2} C_{1}+\alpha_{1}^{2} C_{2}}{4 \alpha_{2}^{2} \alpha_{1}^{1}+\alpha_{2}^{1} \alpha_{1}^{2}}, \frac{2 \alpha_{1}^{1} C_{2}-\alpha_{2}^{1} C_{1}}{4 \alpha_{2}^{2} \alpha_{1}^{1}+\alpha_{2}^{1} \alpha_{1}^{2}}\right)
\end{aligned}
$$

Noticeably, $E_{1}$ is boundary equilibrium point. The fixed point $E_{*}$ is a Nash equilibrium point. The fixed point solutions have economic meaning when the following conditions are satisfied:

$$
\left\{\begin{aligned}
C_{1} & \geq 0 \\
2 \alpha_{1}^{1} C_{2} & \geq \alpha_{2}^{1} C_{1} .
\end{aligned}\right.
$$

In order to study the stability of the fixed points of the three-dimensional system (16), we compute eigenvalues of the Jacobian matrix of this system.

Lemma 1: The boundary equilibrium $E_{1}$ is an unstable solution.

Proof: A detailed proof is available in our technical report in [13].
1) The stability of Nash equilibrium: In order to study the stability of Nash equilibrium $E_{*}$ of the system (15), We estimate the Jacobian matrix $J$ at $E_{*}$ which is:

$$
J\left(E_{*}\right)=\left[\begin{array}{ccc}
0 & 1 & 0 \\
\frac{\alpha_{1}^{2} \alpha_{2}^{1}\left(1-\omega_{1}\right)}{2 \alpha_{1}^{1}} B & \frac{\alpha_{1}^{2} \alpha_{2}^{1} \omega_{1}}{2 \alpha_{1}^{1}} B & \frac{\alpha_{1}^{2}}{2 \alpha_{1}^{1}}\left(1+2 \alpha_{2}^{2} B\right) \\
\alpha_{2}^{1}\left(1-\omega_{1}\right) B & \alpha_{2}^{1} \omega_{1} B & 1+2 \alpha_{2}^{2} B
\end{array}\right]
$$

where $B=\frac{\theta_{2}\left(-2 \alpha_{1}^{1} C_{2}+\alpha_{2}^{1} C_{1}\right)}{\left(4 \alpha_{2}^{2} \alpha_{1}^{1}+\alpha_{2}^{1} \alpha_{1}^{2}\right)}$,

We consider that $P_{3}(\lambda)$ is the characteristic polynomial of $J\left(E_{*}\right)$, so, eigenvalues of Nash equilibrium correspond to the roots of $P_{3}(\lambda)=0$. The Nash equilibrium is stable if the necessary and sufficient condition for the roots of the polynomial $P_{3}(\lambda)$ to satisfy $|\lambda|<1$ can be obtained by applying Jury's test [12]. The characteristic polynomial has the form:

$$
P_{3}(\lambda):=\lambda^{3}+a \lambda^{2}+b \lambda+c=0,
$$

where: $a=-\operatorname{tr}\left(J\left(E_{*}\right)\right), b=\operatorname{tr}\left(\operatorname{com}\left(J\left(E_{*}\right)\right)\right)$ and $c=$ $-\operatorname{det}\left(J\left(E_{*}\right)\right)$ in other word:

$$
\begin{aligned}
& a=-1-\frac{\alpha_{1}^{2} \alpha_{2}^{1} \omega_{1}}{2 \alpha_{1}^{1}} B-2 \alpha_{2}^{2} B \\
& b=-\frac{\alpha_{1}^{2} \alpha_{2}^{1}\left(1-\omega_{1}\right)}{2 \alpha_{1}^{1}} B \\
& c=-\operatorname{det}\left(J\left(E_{*}\right)\right)=0
\end{aligned}
$$

where $\operatorname{com}(\cdot)$ denotes the comatrice operator. Recalling Jury's test [12] for stability of Nash equilibrium, we get the necessary and sufficient conditions for $\left|\lambda_{i}\right|<1, \quad i=1,2,3$ : (i)

$$
\begin{aligned}
& \text { 1) } 1+a+b+c=\frac{2 \alpha_{1}^{1} C_{2}-\alpha_{2}^{1} C_{1}}{2 \alpha_{1}^{1}} \theta_{2}>0 \\
& \text { 2) } 1-a+b-c=2+B\left(2 \alpha_{2}^{2}+\frac{\alpha_{2}^{1} \alpha_{1}^{2}\left(2 \omega_{1}-1\right)}{2 \alpha_{1}^{1}}\right)>0 \text {, } \\
& \text { 3) } 1-c^{2}>|b-a c| \text {, ie } 1>\left|-\frac{\alpha_{1}^{2} \alpha_{2}^{1}\left(1-\omega_{1}\right)}{2 \alpha_{1}^{1}} B\right| \\
& \text { 4) }|c|<1
\end{aligned}
$$

The Nash equilibrium point is locally asymptotically stable if the condition equations (i) to (iv) are satisfied. Moreover, when $\omega_{1}$ is sufficiently small the Nash equilibrium point $E_{*}$ is stable. The stability region with respect to delay $\omega_{1}$ is defined by both conditions (ii) and (iii). Hence, we deduced from above analysis that delay has a stabilization effect for the Nash equilibrium point.

2) Numerical Simulations: The objective of these Numerical experiments is to show that the delay has the effect of increasing the stability domain for system (16). In order to study the local stability properties of the equilibrium points it is convenient to take the parameters' values in Table 1:

\begin{tabular}{|c|c|c|c|c|}
\hline$\alpha_{1}^{1}=\alpha_{2}^{2}$ & $\alpha_{2}^{1}=\alpha_{1}^{2}$ & $\beta_{1}^{1}=\beta_{2}^{2}$ & $\beta_{2}^{1}=\beta_{1}^{2}$ & $D_{0}^{1}=D_{0}^{2}$ \\
\hline 0.7 & 0.3 & 0.7 & 0.3 & 20 \\
\hline \hline $\bar{q}_{1}=\bar{q}_{2}$ & $\bar{g}_{1}=\bar{g}_{2}$ & $\vartheta_{1}=\vartheta_{2}$ & $\omega_{1}$ & $\theta_{2}$ \\
\hline 5 & 10 & 1 & 0.5 & $0<\theta_{2}<0.1$ \\
\hline
\end{tabular}

TABLE I

PARAMETERS' VALUES USED FOR NUMERICAL SIMULATIONS.

Figure 1 plots the bifurcation diagrams of $p_{i}$ with respect to $\theta_{2}$, it shows that the trajectories converge to the equilibrium 
$(28.78,18.62)$ for $\theta_{2}<0.073$. By cons, when $\theta_{2}>0.073$ the Nash equilibrium becomes unstable, period doubling bifurcation appears at $\theta_{2}=0.073$ and finally chaotic behavior occurs.

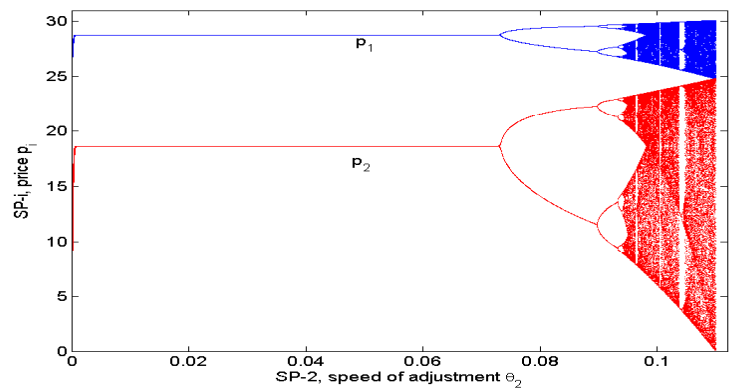

Fig. 1. Bifurcation diagrams of $p_{1}$ (blue) and $p_{2}$ (red) with respect to $\theta_{2}$ in delay case $\left(\omega_{1}=0.5\right)$

Figure 2 and 3 show respectively the bifurcation diagrams of $p_{1}$ and $p_{2}$ with respect to $\theta_{2}$ in two cases: non-delay $\left(\omega_{1}=1\right.$, blue graph) and delay ( $\omega_{1}=0.5$, red graph). Comparing the bifurcation diagrams (blue and red) in each Figure (2 and 3 ), it is observed that period doubling bifurcations in delay case is delayed w.r.t $\theta_{2}$, this result is what we expected.

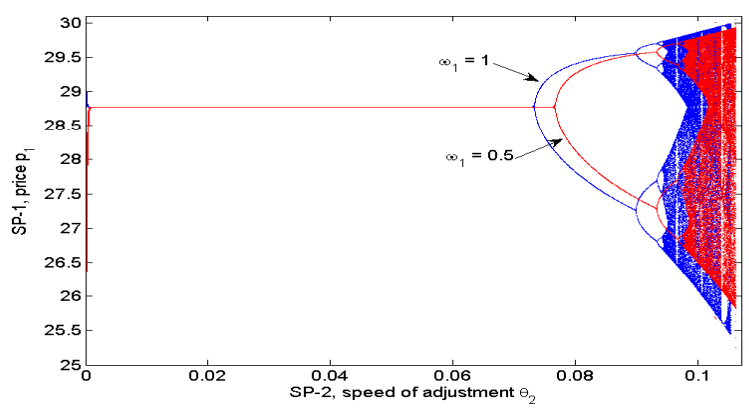

Fig. 2. Bifurcation diagrams of $p_{1}$ with respect to $\theta_{2}$ in two cases: non-delay (blue) and delay (red).

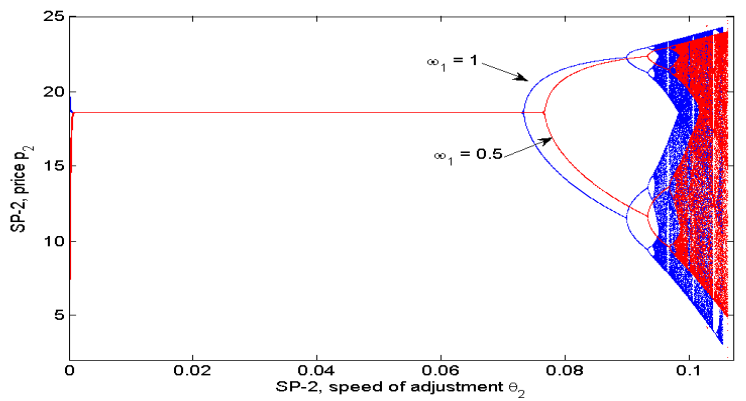

Fig. 3. Bifurcation diagrams of $p_{2}$ with respect to $\theta_{2}$ in two cases: non-delay (blue) and delay (red).

Depending on the size of memory, $T$, Figure 4 plots the maximum value of $\theta_{2}^{\max }$ which guarantees the stability of the equilibrium (point doubling bifurcation). We note that $\theta_{2}^{\max }$ increases with respect to size of memory, this result shows that the size of memory (delay) has the effect of increasing the stability domain for system (16).

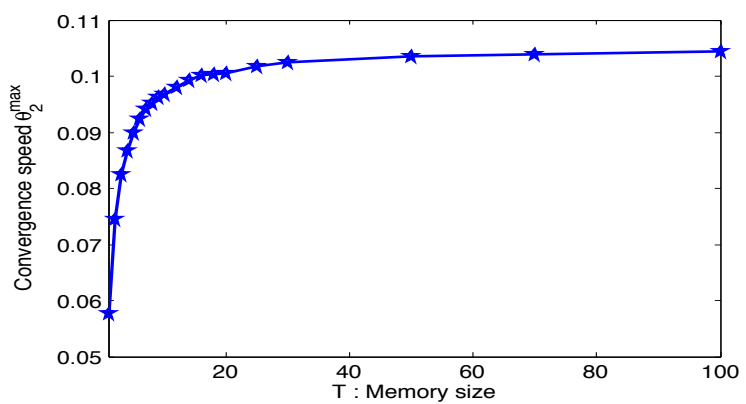

Fig. 4. Convergence speed $\theta_{2}^{\max }$, of price game with respect to memory size of rational SP.

\section{B. Symmetric Scenario}

As explained above, in symmetric scenario, we consider that both SPs are boundedly rational, and as pointed out by Bischi and Naimzada [9], a bounded rational players modify their output decisions dynamically according to their marginal profit: $\frac{\partial \Pi_{i}(\mathbf{p})}{\partial p_{i}}, \quad i \in\{1,2\}$. So, the dynamical system for decisions of SPs has the form:

$$
\left\{\begin{array}{l}
p_{1}(t+1)=p_{1}(t)+\theta_{1} p_{1}(t) \frac{\partial \Pi_{1}\left(p_{1}^{e}(t+1), p_{2}^{e}(t+1)\right)}{\partial p_{1}}, \\
p_{2}(t+1)=p_{2}(t)+\theta_{2} p_{2}(t) \frac{\partial \Pi_{2}\left(p_{1}^{e}(t+1), p_{2}^{e}(t+1)\right)}{\partial p_{2}} .
\end{array}\right.
$$

Where $p_{i}^{e}(t+1)$ is the expected price of SP- $i$ in the next time step $(t+1)$, as explained above, and if we consider that $T=1$, the expected price $p_{i}^{e}(t+1)$ is given by:

$$
p_{i}^{e}(t+1)=\omega_{i} p_{i}(t)+\left(1-\omega_{i}\right) p_{i}(t-1),
$$

so, the dynamical model (19) becomes:

$$
\left\{\begin{aligned}
p_{1}(t+1)= & p_{1}(t)+\theta_{1} p_{1}(t)\left[-2 \alpha_{1}^{1} p_{1}(t)+C_{1}\right. \\
& \left.+\alpha_{1}^{2}\left(\omega_{2} p_{2}(t)+\left(1-\omega_{2}\right) p_{2}(t-1)\right)\right], \\
p_{2}(t+1)= & p_{2}(t)+\theta_{2} p_{2}(t)\left[-2 \alpha_{2}^{2} p_{2}(t)+C_{2}\right. \\
& \left.+\alpha_{2}^{1}\left(\omega_{1} p_{1}(t)+\left(1-\omega_{1}\right) p_{1}(t-1)\right)\right] .
\end{aligned}\right.
$$

In order to study the dynamical system (20), we rewrite it as a fourth dimensional system in the form:

$$
\left\{\begin{aligned}
r_{1}(t+1)= & p_{1}(t) \\
r_{2}(t+1)= & p_{2}(t) \\
p_{1}(t+1)= & p_{1}(t)+\theta_{1} p_{1}(t)\left[-2 \alpha_{1}^{1} p_{1}(t)+C_{1}\right. \\
& \left.+\alpha_{1}^{2}\left(\omega_{2} p_{2}(t)+\left(1-\omega_{2}\right) r_{2}(t)\right)\right], \\
p_{2}(t+1)= & p_{2}(t)+\theta_{2} p_{2}(t)\left[-2 \alpha_{2}^{2} p_{2}(t)+C_{2}\right. \\
& \left.+\alpha_{2}^{1}\left(\omega_{1} p_{1}(t)+\left(1-\omega_{1}\right) r_{1}(t)\right)\right] .
\end{aligned}\right.
$$

The steady states of system (21) are:

$$
\begin{aligned}
& E_{1}=(0,0,0,0) \\
& E_{2}=\left(0, \frac{C_{2}}{2 \alpha_{2}^{2}}, 0, \frac{C_{2}}{2 \alpha_{2}^{2}}\right) \\
& E_{3}=\left(\frac{C_{1}}{2 \alpha_{1}^{1}}, 0, \frac{C_{1}}{2 \alpha_{1}^{1}}, 0\right) \\
& E_{*}=\left(\frac{2 \alpha_{2}^{2} C_{1}-\alpha_{1}^{2} C_{2}}{4 \alpha_{2}^{2} \alpha_{1}^{1}-\alpha_{2}^{1} \alpha_{1}^{2}}, \frac{2 \alpha_{1}^{1} C_{2}-\alpha_{2}^{1} C_{1}}{4 \alpha_{2}^{2} \alpha_{1}^{1}-\alpha_{2}^{1} \alpha_{1}^{2}}, \frac{2 \alpha_{2}^{2} C_{1}-\alpha_{1}^{2} C_{2}}{4 \alpha_{2}^{2} \alpha_{1}^{1}-\alpha_{2}^{1} \alpha_{1}^{2}}, \frac{2 \alpha_{1}^{1} C_{2}-\alpha_{2}^{1} C_{1}}{4 \alpha_{2}^{2} \alpha_{1}^{1}-\alpha_{2}^{1} \alpha_{1}^{2}}\right)
\end{aligned}
$$

where $E_{1}, E_{2}$ and $E_{3}$ are the boundary equilibria. The equilibrium $E_{*}$ is called Nash equilibrium. From Assumption (1) we deduce directly that $4 \alpha_{2}^{2} \alpha_{1}^{1}-\alpha_{2}^{1} \alpha_{1}^{2}>0$, so, fixed points 
of the dynamical system (21) have economic meaning when the following conditions are satisfied:

$$
\left\{\begin{array}{ccc}
C_{1}>0 & \text { and } & C_{2}>0 \\
2 \alpha_{1}^{1} C_{2} & > & \alpha_{2}^{1} C_{1} \\
2 \alpha_{2}^{2} C_{1} & > & \alpha_{1}^{2} C_{2}
\end{array}\right.
$$

Lemma 2: The boundary equilibria $E_{1}, E_{2}$ and $E_{3}$ are unstable solutions. in [13].

Proof: A detailed proof is available in our technical report

1) The stability of Nash equilibrium: At Nash equilibrium $E_{*}$, the Jacobian matrix is:

$J=\left[\begin{array}{cccc}0 & 0 & 1 & 0 \\ 0 & 0 & 0 & 1 \\ 0 & \alpha_{1}^{2} G_{1}\left(1-\omega_{2}\right) & 1+2 \alpha_{1}^{1} G_{1} & \alpha_{1}^{2} G_{1} \omega_{2} \\ \alpha_{2}^{1} G_{2}\left(1-\omega_{1}\right) & 0 & \alpha_{2}^{1} G_{2} \omega_{1} & 1+2 \alpha_{2}^{2} G_{2}\end{array}\right]$ where $G_{1}=\frac{\theta_{1}\left(-2 \alpha_{2}^{2} C_{1}+\alpha_{1}^{2} C_{2}\right)}{\left(4 \alpha_{2}^{2} \alpha_{1}^{1}-\alpha_{2}^{1} \alpha_{1}^{2}\right)}$ and $G_{2}=\frac{\theta_{2}\left(-2 \alpha_{1}^{1} C_{2}+\alpha_{2}^{1} C_{1}\right)}{\left(4 \alpha_{2}^{2} \alpha_{1}^{1}-\alpha_{2}^{1} \alpha_{1}^{2}\right)}$.

Stability conditions of Nash equilibrium $E_{*}$ are that all root of the equation $P_{4}(\lambda)=0$ satisfy $|\lambda|>1$, where $P_{4}(\lambda)$ is the characteristic polynomial of Jacobian matrix in Nash equilibrium point :

$$
P_{4}(\lambda)=\lambda^{4}+a \lambda^{3}+b \lambda^{2}+c \lambda+d,
$$

such that:

$$
\begin{aligned}
& a=-2\left(1+\alpha_{1}^{1} G_{1}+\alpha_{2}^{2} G_{2}\right) \\
& b=1+2 \alpha_{1}^{1} G_{1}+2 \alpha_{2}^{2} G_{2}+G_{1} G_{2}\left(4 \alpha_{1}^{1} \alpha_{2}^{2}-\alpha_{1}^{2} \alpha_{2}^{1} \omega_{1} \omega_{2}\right) \\
& c=-\alpha_{1}^{2} \alpha_{2}^{1} G_{1} G_{2}\left(\omega_{1}-2 \omega_{1} \omega_{2}+\omega_{2}\right) \\
& d=-\alpha_{1}^{2} \alpha_{2}^{1} G_{1} G_{2}\left(1-\omega_{1}-\omega_{2}+\omega_{1} \omega_{2}\right) .
\end{aligned}
$$

According the Jury criteria, [12], the Nash equilibrium point is locally asymptotically stable if the following condition equations are satisfied : (i)

1) $1+a+b+c+d>0$,

2) $1-a+b-c+d>0$,

3) $(1-d)\left(1-d^{4}\right)-b(1-d)^{2}+(a-c)(c-a d)>0$

4) $3+3 d>b$

5) $|d|<1$.

2) Numerical Simulations: In this numerical experiments, we used the same parameters' values considered in Table 1. Bifurcation diagrams are drawn in Figure 5, we see that duopoly model with bounded rationality $\left(\omega_{1}=\omega_{2}=0.5\right)$ is stable for $\theta_{2}<0.068$ and $\theta_{1}=0.05$, period doubling bifurcation appears at $\theta_{2}=0.068$ and finally chaotic behavior occurs.

Figure 6 shows the bifurcation diagrams of $p_{1}$ with respect to $\theta_{1}$ when $\theta_{2}=0.05$ in two cases : non-delay case $\left(\omega_{1}=\omega_{2}=1\right.$, blue graph $)$ and delay case $\left(\omega_{1}=\omega_{2}=0.5\right.$, red graph). Comparing the bifurcation diagrams (blue and red), it is clear that period doubling bifurcations in delay case is delayed w.r.t $\theta_{1}$, so, we deduced that delay has a stabilization effect for the Nash equilibrium point. Figure 7 plots the bifurcation diagrams of $p_{1}$ w.r.t $\omega_{1}=\omega_{2}$. From this Figure we deduce that the delay increases the stability domain, i.e when $\omega_{1}$ and $\omega_{2}$ are sufficiently small (less than 0.33 in this parameters' values example), the Nash equilibrium point is stable.

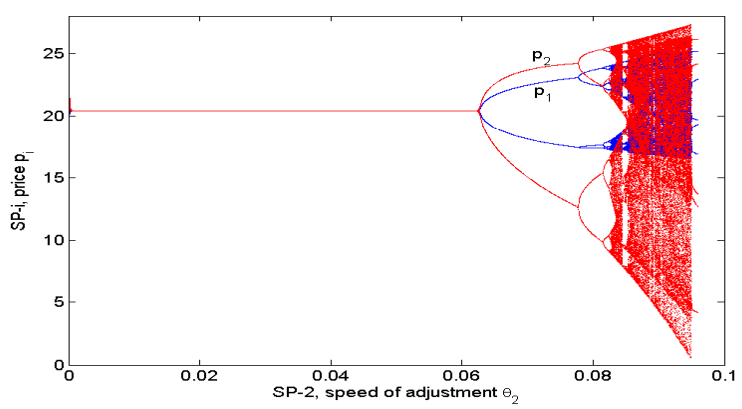

Fig. 5. Bifurcation diagrams of $p_{1}$ and $p_{2}$ with respect to $\theta_{2}$ in delay case $\left(\omega_{1}=\omega_{2}=0.5\right)$, when $\theta_{1}=0.05$.

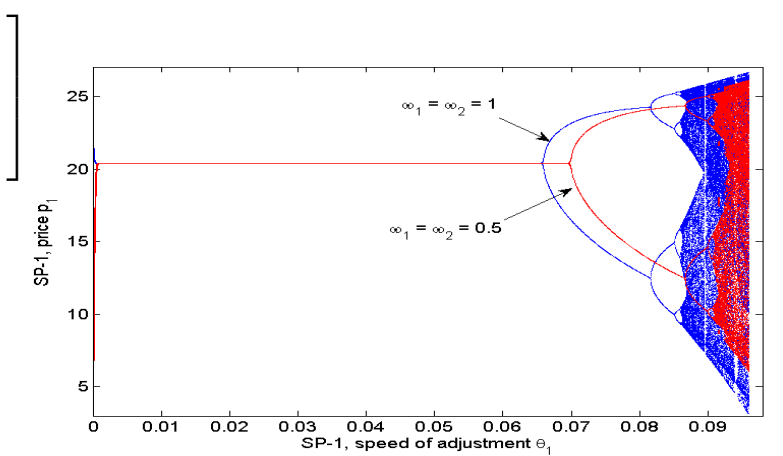

Fig. 6. Bifurcation diagrams of $p_{1}$ with respect to $\theta_{1}$ in two cases: non-delay (blue) and delay (red), when $\theta_{2}=0.05$.

\section{JOINT PRICE AND QOS GAME}

We consider simultaneous setting of both parameters by all SPs, the joint price/QoS game arises when the SP- $i$ decides on his both price and QoS in order to maximize his profit. Considering the bounded rationality duopoly game, the profit resulting at the discrete time, $t$, for $\mathrm{SP}-i, \quad i \in\{1,2\}$, is given by :

$\Pi_{i}(t)=D_{i}(t)\left(p_{i}(t)-\vartheta_{i} g_{i}\left(q_{i}(t)\right)\right)-\vartheta_{i} h_{i}\left(q_{i}(t)\right), \quad \forall i \in\{1,2\}$,

where $D_{i}(t)=D_{i}(\mathbf{p}(t), \mathbf{q}(t))$.

We suppose that the measure defining the QoS, $q_{i}$ corresponds to some function of the expected delay. We shall use the Kleinrock delay function ${ }^{1}$ which is a common delay function used in networking games [4]. So : $q_{i}=\frac{1}{\sqrt{\text { Delay }_{i}}}=$ $\sqrt{\mu_{i}-D_{i}}$, that mean : $\mu_{i}\left(q_{i}, D_{i}\right)=q_{i}^{2}+D_{i}$, from this latter and according to equation (4), we have that $g_{i}\left(q_{i}\right)=1$ and $h_{i}\left(q_{i}\right)=q_{i}^{2}$, so, equation (23) become:

$$
\Pi_{i}(t)=D_{i}(t)\left(p_{i}(t)-\vartheta_{i}\right)-\vartheta_{i}\left(q_{i}(t)\right)^{2}, \quad \forall i \in\{1,2\},
$$

As pointed out above (Section III), a bounded rational player $\mathrm{SP} i$ modifies its output decisions (price, $p_{i}$, and QoS, $q_{i}$ ) according respectively to its marginal profits : $\frac{\partial \Pi_{i}(\mathbf{p}, \mathbf{q})}{\partial p_{i}}$ and $\frac{\partial \Pi_{i}(\mathbf{p}, \mathbf{q})}{\partial q_{i}}$. Therefore, the dynamical equation for decisions of

\footnotetext{
${ }^{1}$ This function corresponds to the queuing delay in an $M / M / 1$ queue with first-in-first-out discipline or to the more general $M / G / 1$ queue under processor sharing delay.
} 


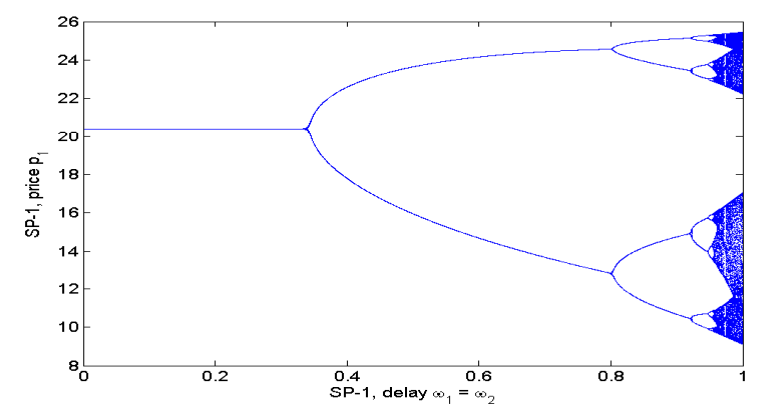

Fig. 7. Bifurcation diagrams of $p_{1}$ with respect to $\omega_{1}=\omega_{2}$.

$\mathrm{SP}-i$ has the form:

$$
\left\{\begin{aligned}
p_{i}(t+1) & =p_{i}(t)+\theta_{i} p_{i}(t) \frac{\partial \Pi_{i}\left(\mathbf{p}^{D}, \mathbf{q}^{D}\right)}{\partial p_{i}} \\
q_{i}(t+1) & =q_{i}(t)+\rho_{i} q_{i}(t) \frac{\partial \Pi_{i}\left(\mathbf{p}^{D}, \mathbf{q}^{D}\right)}{\partial q_{i}}
\end{aligned}\right.
$$

where, $\theta_{i}$ and $\rho_{i}$ represent respectively the speed of adjustment of price and QoS of $i^{t h} \mathrm{SP}$, and $p^{D}=\left(p_{i}(t), p_{j}^{e}(t+1)\right)$, $q^{D}=\left(q_{i}(t), q_{j}^{e}(t+1)\right)$, such that $p_{j}^{e}(t+1)$ and $p_{j}^{e}(t+1)$ are respectively the expected price and QoS of the opponent in the next time step, $(t+1)$.

As explained above, and if we consider that $T=1$, the expected price $p_{j}^{e}(t+1)$ and the expected QoS $q_{j}^{e}(t+1)$ are given by:

$$
\left\{\begin{array}{l}
p_{j}^{e}(t+1)=\omega_{j} p_{j}(t)+\left(1-\omega_{j}\right) p_{j}(t-1) \\
q_{j}^{e}(t+1)=\nu_{j} q_{j}(t)+\left(1-\nu_{j}\right) q_{j}(t-1)
\end{array}\right.
$$

where the positive constants $\omega_{j}$ and $\nu_{j}$ represent respectively the weights given to previous prices and QoSs of $S P_{j}$.

The marginal profits of the ith SP respectively at the point $\left(p_{1}, p_{2}\right)$ and $\left(q_{1}, q_{2}\right)$ of the strategy space are :

$$
\left\{\begin{array}{l}
\frac{\partial \Pi_{i}}{\partial p_{i}}=-\alpha_{i}^{i}\left(p_{i}-\vartheta_{i}\right)+D_{i} \quad i=1,2 . \\
\frac{\partial \Pi_{i}}{\partial q_{i}}=\beta_{i}^{i}\left(p_{i}-\vartheta_{i}\right)-2 \vartheta_{i} q_{i} \quad i=1,2 .
\end{array}\right.
$$

So, the dynamical model (25) becomes:

$$
\left\{\begin{aligned}
p_{1}(t+1)= & p_{1}(t)+\theta_{1} p_{1}(t)\left[D_{1}^{0}+\vartheta_{1} \alpha_{1}^{1}-2 \alpha_{1}^{1} p_{1}(t)+\beta_{1}^{1} q_{1}(t)\right. \\
& +\alpha_{1}^{2}\left(\omega_{2} p_{2}(t)+\left(1-\omega_{2}\right) p_{2}(t-1)\right)-\beta_{1}^{2}\left(\nu_{2} q_{2}(t)\right. \\
& \left.\left.+\left(1-\nu_{2}\right) q_{2}(t-1)\right)\right] \\
p_{2}(t+1)= & p_{2}(t)+\theta_{2} p_{2}(t)\left[D_{2}^{0}+\vartheta_{2} \alpha_{2}^{2}-2 \alpha_{2}^{2} p_{2}(t)+\beta_{2}^{2} q_{2}(t)\right. \\
& +\alpha_{2}^{1}\left(\omega_{1} p_{1}(t)+\left(1-\omega_{1}\right) p_{1}(t-1)\right)-\beta_{2}^{1}\left(\nu_{1} q_{1}(t)\right. \\
& \left.\left.+\left(1-\nu_{1}\right) q_{1}(t-1)\right)\right] \\
q_{1}(t+1)= & q_{1}(t)+\rho_{1} q_{1}(t)\left[-2 \vartheta_{1} q_{1}(t)+\beta_{1}^{1} p_{1}(t)-\beta_{1}^{1} \vartheta_{1}\right] \\
q_{2}(t+1)= & q_{2}(t)+\rho_{2} q_{2}(t)\left[-2 \vartheta_{2} q_{2}(t)+\beta_{2}^{2} p_{2}(t)-\beta_{2}^{2} \vartheta_{2}\right]
\end{aligned}\right.
$$

To study stability of (26) we rewrite it as a eight-dimensional system in the form:

$$
\left\{\begin{aligned}
r_{1}(t+1)= & p_{1}(t) \\
r_{2}(t+1)= & p_{2}(t) \\
s_{1}(t+1)= & q_{1}(t) \\
s_{2}(t+1)= & q_{2}(t) \\
p_{1}(t+1)= & p_{1}(t)+\theta_{1} p_{1}(t)\left[D_{1}^{0}+\vartheta_{1} \alpha_{1}^{1}-2 \alpha_{1}^{1} p_{1}(t)+\beta_{1}^{1} q_{1}(t)\right. \\
& +\alpha_{1}^{2}\left(\omega_{2} p_{2}(t)+\left(1-\omega_{2}\right) r_{2}(t)\right)-\beta_{1}^{2}\left(\nu_{2} q_{2}(t)\right. \\
& \left.\left.+\left(1-\nu_{2}\right) s_{2}(t)\right)\right] \\
p_{2}(t+1)= & p_{2}(t)+\theta_{2} p_{2}(t)\left[D_{2}^{0}+\vartheta_{2} \alpha_{2}^{2}-2 \alpha_{2}^{2} p_{2}(t)+\beta_{2}^{2} q_{2}(t)\right. \\
& +\alpha_{2}^{1}\left(\omega_{1} p_{1}(t)+\left(1-\omega_{1}\right) r_{1}(t)\right)-\beta_{2}^{1}\left(\nu_{1} q_{1}(t)\right. \\
& \left.\left.+\left(1-\nu_{1}\right) s_{1}(t)\right)\right] \\
q_{1}(t+1)= & q_{1}(t)+\rho_{1} q_{1}(t)\left[-2 \vartheta_{1} q_{1}(t)+\beta_{1}^{1} p_{1}(t)-\beta_{1}^{1} \vartheta_{1}\right] \\
q_{2}(t+1)= & q_{2}(t)+\rho_{2} q_{2}(t)\left[-2 \vartheta_{2} q_{2}(t)+\beta_{2}^{2} p_{2}(t)-\beta_{2}^{2} \vartheta_{2}\right]
\end{aligned}\right.
$$

There exists sixteen fixed points of (27):

$$
\begin{aligned}
& E_{1}=(0,0,0,0,0,0,0,0) \\
& E_{2}=\left(0,0,0, \frac{-C_{2}^{q}}{2 \vartheta_{2}}, 0,0,0, \frac{-C_{2}^{q}}{2 \vartheta_{2}}\right) \\
& E_{3}=\left(0,0, \frac{-C_{1}^{q}}{2 \vartheta_{1}}, 0,0,0, \frac{-C_{1}^{q}}{2 \vartheta_{1}}, 0\right) \\
& E_{4}=\left(0,0, \frac{-C_{1}^{q}}{2 \vartheta_{1}}, \frac{-C_{2}^{q}}{2 \vartheta_{2}}, 0,0, \frac{-C_{1}^{q}}{2 \vartheta_{1}}, \frac{-C_{2}^{q}}{2 \vartheta_{2}}\right) \\
& E_{5}=\left(0, \frac{C_{2}^{p}}{2 \alpha_{2}^{2}}, 0,0,0, \frac{C_{2}^{p}}{2 \alpha_{2}^{2}}, 0,0\right) \\
& E_{6}=\left(0, r_{2}^{6}, 0, s_{2}^{6}, 0, p_{2}^{6}, 0, q_{2}^{6}\right) \\
& E_{7}=\left(0, \frac{2 C_{2}^{p} \vartheta_{1}-\beta_{2}^{1} C_{1}^{q}}{4 \vartheta_{1} \alpha_{2}^{2}}, \frac{-C_{1}^{q}}{2 \vartheta_{1}}, 0,0, \frac{2 C_{2}^{p} \vartheta_{1}-\beta_{2}^{1} C_{1}^{q}}{4 \vartheta_{1} \alpha_{2}^{2}}, \frac{-C_{1}^{q}}{2 \vartheta_{1}}, 0\right) \\
& E_{8}=\left(0, r_{2}^{8}, s_{1}^{8}, s_{2}^{8}, 0, p_{2}^{8}, q_{1}^{8}, q_{2}^{8}\right) \\
& E_{9}=\left(\frac{C_{1}^{p}}{2 \alpha_{1}^{1}}, 0,0,0, \frac{C_{1}^{p}}{2 \alpha_{1}^{1}}, 0,0,0\right) \\
& E_{10}=\left(\frac{2 C_{1}^{p} \vartheta_{2}-\beta_{1}^{2} C_{2}^{q}}{4 \vartheta_{2} \alpha_{1}^{1}}, 0,0, \frac{-C_{2}^{q}}{2 \vartheta_{2}}, \frac{2 C_{1}^{p} \vartheta_{2}-\beta_{1}^{2} C_{2}^{q}}{4 \vartheta_{2} \alpha_{1}^{1}}, 0,0, \frac{-C_{2}^{q}}{2 \vartheta_{2}}\right) \\
& E_{11}=\left(r_{1}^{11}, 0, s_{1}^{11}, s_{2}^{11}, p_{1}^{11}, 0, q_{1}^{11}, q_{2}^{11}\right) \\
& E_{12}=\left(r_{1}^{12}, 0, s_{1}^{12}, 0, p_{1}^{12}, 0, q_{1}^{12}, 0\right) \\
& E_{13}=\left(r_{1}^{13}, r_{2}^{13}, 0,0, p_{1}^{13}, p_{2}^{13}, 0,0\right) \\
& E_{14}=\left(r_{1}^{14}, r_{2}^{14}, 0, s_{2}^{14}, p_{1}^{14}, p_{2}^{14}, 0, q_{2}^{14}\right) \\
& E_{15}=\left(r_{1}^{15}, r_{2}^{15}, s_{1}^{15}, 0, p_{1}^{15}, p_{2}^{15}, q_{1}^{15}, 0\right) \\
& E_{*}=\left(r_{1}^{*}, r_{2}^{*}, s_{1}^{*}, s_{2}^{*}, p_{1}^{*}, p_{2}^{*}, q_{1}^{*}, q_{2}^{*}\right)
\end{aligned}
$$

such that:

$$
\begin{aligned}
C_{i}^{p} & =D_{i}^{0}+\vartheta_{i} \alpha_{i}^{i}, \quad C_{i}^{q}=\beta_{i}^{i} \vartheta_{i} \\
r_{2}^{6} & =p_{2}^{6}=\frac{2 C_{2}^{p} \vartheta_{2}-\beta_{2}^{2} C_{2}^{q}}{4 \vartheta_{2} \alpha_{2}^{2}+\left(\beta_{2}^{2}\right)^{2}}, \quad s_{2}^{6}=q_{2}^{6}=\frac{\beta_{2}^{2} C_{2}^{p}-2 C_{2}^{q} \alpha_{2}^{2}}{4 \vartheta_{2} \alpha_{2}^{2}+\left(\beta_{2}^{2}\right)^{2}} \\
r_{2}^{8} & =p_{2}^{8}=\frac{2 C_{2}^{p} \vartheta_{1} \vartheta_{2}-\beta_{2}^{2} C_{2}^{q} \vartheta_{1}+\beta_{2}^{1} C_{1}^{q} \vartheta_{2}}{\vartheta_{1}\left(4 \vartheta_{2} \alpha_{2}^{2}+\left(\beta_{2}^{2}\right)^{2}\right)}, \quad s_{1}^{8}=q_{1}^{8}=\frac{-C_{1}^{q}}{2 \vartheta_{1}}, \\
\left(_{26}\right)_{2}^{8} & =q_{2}^{8}=\frac{2 \beta_{2}^{2} C_{2}^{p} \vartheta_{1}+\beta_{2}^{2} \beta_{2}^{1} C_{1}^{q}-4 C_{2}^{q} \alpha_{2}^{2} \vartheta_{1}}{2 \vartheta_{1}\left(4 \vartheta_{2} \alpha_{2}^{2}+\left(\beta_{2}^{2}\right)^{2}\right)}, \\
r_{1}^{11} & =p_{1}^{11}=\frac{2 \vartheta_{1} \vartheta_{2} C_{1}^{p}+\vartheta_{1} C_{2}^{q} \beta_{1}^{2}-C_{1}^{q} \vartheta_{2} \beta_{1}^{1}}{\vartheta_{2}\left(4 \vartheta_{1} \alpha_{1}^{1}+\left(\beta_{1}^{1}\right)^{2}\right)}, \quad s_{2}^{11}=q_{2}^{11}=\frac{-C_{2}^{q}}{2 \vartheta_{2}}, \\
s_{1}^{11} & =q_{1}^{11}=\frac{2 \vartheta_{2} \beta_{1}^{1} C_{1}^{p}-4 \vartheta_{2} C_{1}^{q} \alpha_{1}^{1}+C_{2}^{q} \beta_{1}^{1} \beta_{1}^{2}}{2 \vartheta_{2}\left(4 \vartheta_{1} \alpha_{1}^{1}+\left(\beta_{1}^{1}\right)^{2}\right)},
\end{aligned}
$$




$$
\begin{aligned}
& r_{1}^{12}=p_{1}^{12} \\
&=\frac{2 \vartheta_{1} C_{1}^{p}-C_{1}^{q} \beta_{1}^{1}}{4 \vartheta_{1} \alpha_{1}^{1}+\left(\beta_{1}^{1}\right)^{2}}, \quad s_{1}^{12}=q_{1}^{12}=\frac{\beta_{1}^{1} C_{1}^{p}-2 C_{1}^{q} \alpha_{1}^{1}}{4 \vartheta_{1} \alpha_{1}^{1}+\left(\beta_{1}^{1}\right)^{2}} \\
& r_{1}^{13}=p_{1}^{13} \\
&=\frac{2 C_{1}^{p} \alpha_{2}^{2}+\alpha_{1}^{2} C_{2}^{p}}{4 \alpha_{2}^{2} \alpha_{1}^{1}-\alpha_{2}^{1} \alpha_{1}^{2}}, \quad r_{2}^{13}=p_{2}^{13}=\frac{2 C_{2}^{p} \alpha_{1}^{1}+\alpha_{2}^{1} C_{1}^{p}}{4 \alpha_{2}^{2} \alpha_{1}^{1}-\alpha_{2}^{1} \alpha_{1}^{2}} \\
& r_{1}^{14}=p_{1}^{14} \\
&=\frac{\alpha_{1}^{2}\left(2 C_{2}^{p} \vartheta_{2}-\beta_{2}^{2} C_{2}^{q}\right)-\beta_{2}^{2}\left(\beta_{1}^{2} C_{2}^{p}+C_{1}^{p} \beta_{2}^{2}\right)+2 \alpha_{2}^{2}\left(C_{2}^{q} \beta_{1}^{2}+2 \vartheta_{2} C_{1}^{p}\right)}{\beta_{2}^{2}\left(-2 \beta_{2}^{2} \alpha_{1}^{1}+\alpha_{2}^{1} \beta_{1}^{2}\right)+2 \vartheta_{2}\left(4 \alpha_{2}^{2} \alpha_{1}^{1}-\alpha_{2}^{1} \alpha_{1}^{2}\right)} \\
& r_{2}^{14}=p_{2}^{14} \\
&=\frac{2 \alpha_{1}^{1}\left(2 C_{2}^{p} \vartheta_{2}-\beta_{2}^{2} C_{2}^{q}\right)+\alpha_{2}^{1}\left(2 C_{1}^{p} \vartheta_{2}+\beta_{1}^{2} C_{2}^{q}\right)}{\beta_{2}^{2}\left(-2 \beta_{2}^{2} \alpha_{1}^{1}+\alpha_{2}^{1} \beta_{1}^{2}\right)+2 \vartheta_{2}\left(4 \alpha_{2}^{2} \alpha_{1}^{1}-\alpha_{2}^{1} \alpha_{1}^{2}\right)} \\
& s_{2}^{14}=q_{2}^{14} \\
&=\frac{\beta_{2}^{2}\left(2 C_{2}^{p} \alpha_{1}^{1}+\alpha_{2}^{1} C_{1}^{p}\right)+C_{2}^{q}\left(-4 \alpha_{2}^{2} \alpha_{1}^{1}+\alpha_{2}^{1} \alpha_{1}^{2}\right)}{\beta_{2}^{2}\left(-2 \beta_{2}^{2} \alpha_{1}^{1}+\alpha_{2}^{1} \beta_{1}^{2}\right)+2 \vartheta_{2}\left(4 \alpha_{2}^{2} \alpha_{1}^{1}-\alpha_{2}^{1} \alpha_{1}^{2}\right)} \\
& r_{1}^{15}=p_{1}^{15} \\
&=\frac{2 \vartheta_{1}\left(\alpha_{1}^{2} C_{2}^{p}+2 C_{1}^{p} \alpha_{2}^{2}\right)+C_{1}^{q}\left(\alpha_{1}^{2} \beta_{2}^{1}-2 \beta_{1}^{1} \alpha_{2}^{2}\right)}{\beta_{1}^{1}\left(\alpha_{1}^{2} \beta_{2}^{1}-2 \beta_{1}^{1} \alpha_{2}^{2}\right)+2 \vartheta_{1}\left(4 \alpha_{1}^{1} \alpha_{2}^{2}-\alpha_{2}^{1} \alpha_{1}^{2}\right)} \\
& r_{2}^{15}=p_{2}^{15} \\
&=\frac{2 \alpha_{1}^{1}\left(2 C_{2}^{p} \vartheta_{1}+\beta_{2}^{1} C_{1}^{q}\right)+C_{1}^{p}\left(2 \vartheta_{1} \alpha_{2}^{1}-\beta_{2}^{1} \beta_{1}^{1}\right)-\beta_{1}^{1}\left(C_{2}^{p} \beta_{1}^{1}+C_{1}^{q} \alpha_{2}^{1}\right)}{\beta_{1}^{1}\left(\alpha_{1}^{2} \beta_{2}^{1}-2 \beta_{1}^{1} \alpha_{2}^{2}\right)+2 \vartheta_{1}\left(4 \alpha_{1}^{1} \alpha_{2}^{2}-\alpha_{2}^{1} \alpha_{1}^{2}\right)} \\
& s_{1}^{15}=q_{1}^{15} \\
&=\frac{\beta_{1}^{1}\left(\alpha_{1}^{2} C_{2}^{p}+2 C_{1}^{p} \alpha_{2}^{2}\right)+C_{1}^{q}\left(-4 \alpha_{1}^{1} \alpha_{2}^{2}+\alpha_{2}^{1} \alpha_{1}^{2}\right)}{\beta_{1}^{1}\left(\alpha_{1}^{2} \beta_{2}^{1}-2 \beta_{1}^{1} \alpha_{2}^{2}\right)+2 \vartheta}, \\
& r_{1}\left(4 \alpha_{1}^{1} \alpha_{2}^{2}-\alpha_{2}^{1} \alpha_{1}^{2}\right)
\end{aligned}
$$

Since $C_{i}^{q}$ is a positive constant, then fixed points: $E_{2}, E_{3}, E_{4}$, $E_{7}, E_{8}, E_{10}$ and $E_{11}$ have at least one negative component. Thus they don't have an economic meaning, this is why we will only consider the following fixed points : $E_{1}, E_{5}, E_{6}$, $E_{9}, E_{12}, E_{13}, E_{14}, E_{15}$ and $E_{*}$. These latter fixed points have economic meaning when all their components are positive.

Assumption 3: We consider that the necessary conditions to ensure that fixed points $E_{1}, E_{5}, E_{6}, E_{9}, E_{12}, E_{13}, E_{14}, E_{15}$ and $E_{*}$ have an economic significance are satisfied.

The equilibria $E_{1}, \ldots, E_{15}$ are called boundary equilibria [9], and the equilibrium $E_{*}$ is a unique Nash equilibrium with components:

$$
\begin{aligned}
r_{1}^{*} & =p_{1}^{*} \\
& =\left\{4 \alpha_{2}^{2}\left(\vartheta_{1} C_{2}^{q} \beta_{1}^{2}+2 \vartheta_{1} \vartheta_{2} C_{1}^{p}-C_{1}^{q} \vartheta_{2} \beta_{1}^{1}\right)\right. \\
& +\beta_{2}^{2}\left(-2 \vartheta_{1} \beta_{2}^{2} C_{1}^{p}-2 \vartheta_{1} C_{2}^{p} \beta_{1}^{2}+C_{1}^{q} \beta_{2}^{2} \beta_{1}^{1}-2 \vartheta_{1} C_{2}^{q} \alpha_{1}^{2}\right. \\
& \left.\left.-C_{1}^{q} \beta_{2}^{1} \beta_{1}^{2}\right)+2 \alpha_{1}^{2} \vartheta_{2}\left(2 \vartheta_{1} C_{2}^{p}+C_{1}^{q} \beta_{2}^{1}\right)\right\} \div A, \\
r_{2}^{*} & =p_{2}^{*} \\
& =\left\{4 \alpha_{1}^{1}\left(2 C_{2}^{p} \vartheta_{1} \vartheta_{2}-\beta_{2}^{2} C_{2}^{q} \vartheta_{1}+\beta_{2}^{1} C_{1}^{q} \vartheta_{2}\right)\right. \\
& +\beta_{1}^{1}\left(-2 \beta_{2}^{1} \vartheta_{2} C_{1}^{p}-2 \alpha_{2}^{1} C_{1}^{q} \vartheta_{2}-2 C_{2}^{p} \vartheta_{2} \beta_{1}^{1}+\beta_{1}^{1} C_{2}^{q} \beta_{2}^{2}\right. \\
& \left.\left.-\beta_{2}^{1} C_{2}^{q} \beta_{1}^{2}\right)+2 \alpha_{2}^{1} \vartheta_{1}\left(2 C_{1}^{p} \vartheta_{2}+C_{2}^{q} \beta_{1}^{2}\right)\right\} \div A, \\
s_{1}^{*} & =q_{1}^{*} \\
& =\left\{C_{1}^{q}\left(2 \vartheta_{2} \alpha_{2}^{1} \alpha_{1}^{2}-8 \alpha_{1}^{1} \alpha_{2}^{2} \vartheta_{2}+2 \alpha_{1}^{1}\left(\beta_{2}^{2}\right)^{2}-\beta_{2}^{2} \alpha_{2}^{1} \beta_{1}^{2}\right)\right. \\
& +\beta_{1}^{1}\left(2 \vartheta_{2} \alpha_{1}^{2} C_{2}^{p}+2 \alpha_{2}^{2} C_{2}^{q} \beta_{1}^{2}-\left(\beta_{2}^{2}\right)^{2} C_{1}^{p}+4 \alpha_{2}^{2} \vartheta_{2} C_{1}^{p}\right. \\
& \left.\left.-\beta_{2}^{2} C_{2}^{p} \beta_{1}^{2}-C_{2}^{q} \alpha_{1}^{2} \beta_{2}^{2}\right)\right\} \div A \\
s_{2}^{*} & =q_{2}^{*} \\
& =\left\{C_{2}^{q}\left(2 \alpha_{1}^{2} \vartheta_{1} \alpha_{2}^{1}-\alpha_{1}^{2} \beta_{1}^{1} \beta_{2}^{1}-8 \vartheta_{1} \alpha_{1}^{1} \alpha_{2}^{2}+2\left(\beta_{1}^{1}\right)^{2} \alpha_{2}^{2}\right)\right. \\
& +\beta_{2}^{2}\left(4 \alpha_{1}^{1} \vartheta_{1} C_{2}^{p}+2 \alpha_{1}^{1} C_{1}^{q} \beta_{2}^{1}-\left(\beta_{1}^{1}\right)^{2} C_{2}^{p}-\beta_{1}^{1} C_{1}^{p} \beta_{2}^{1}\right. \\
& \left.\left.-\beta_{1}^{1} C_{1}^{q} \alpha_{2}^{1}+2 C_{1}^{p} \vartheta_{1} \alpha_{2}^{1}\right)\right\} \div A,
\end{aligned}
$$

such that:

$$
\begin{aligned}
A & =2 \vartheta_{1}\left(8 \alpha_{1}^{1} \alpha_{2}^{2} \vartheta_{2}-2 \alpha_{1}^{1}\left(\beta_{2}^{2}\right)^{2}+\beta_{2}^{2} \alpha_{2}^{1} \beta_{1}^{2}\right)+\beta_{2}^{2} \beta_{1}^{1}\left(\beta_{2}^{2} \beta_{1}^{1}-\beta_{2}^{1} \beta_{1}^{2}\right) \\
& +2 \vartheta_{2}\left(-2 \alpha_{2}^{2}\left(\beta_{1}^{1}\right)^{2}+\beta_{1}^{1} \alpha_{1}^{2} \beta_{2}^{1}-2 \vartheta_{1} \alpha_{2}^{1} \alpha_{1}^{2}\right)
\end{aligned}
$$

\section{A. Stability of equilibria}

1) Stability of boundary equilibria: To study the stability of equilibria of the dynamical system (27), we calculate the Jacobian matrix of system (27) at the state variables $\left(r_{1}, r_{2}, s_{1}, s_{2}, p_{1}, p_{2}, q_{1}, q_{2}\right)$.

Lemma 3: The boundary equilibria $E_{1}, E_{5}, E_{6}, E_{9}, E_{12}$, $E_{13}, E_{14}$, and $E_{15}$ are unstable solutions. in [13].

Proof: A detailed proof is available in our technical report

2) Stability of Nash equilibrium: The Jacobian at Nash equilibrium $E_{*}$ is could be written as

$$
J\left(E_{*}\right)=\left[\begin{array}{cccccccc}
0 & 0 & 0 & 0 & 1 & 0 & 0 & 0 \\
0 & 0 & 0 & 0 & 0 & 1 & 0 & 0 \\
0 & 0 & 0 & 0 & 0 & 0 & 1 & 0 \\
0 & 0 & 0 & 0 & 0 & 0 & 0 & 1 \\
0 & F_{1}^{*} & 0 & G_{1}^{*} & B_{1}^{p^{*}} & H_{1}^{*} & L_{1}^{*} & M_{1}^{*} \\
F_{2}^{*} & 0 & G_{2}^{*} & 0 & H_{2}^{*} & B_{2}^{p^{*}} & M_{2}^{*} & L_{2}^{*} \\
0 & 0 & 0 & 0 & N_{1}^{*} & 0 & B_{1}^{q^{*}} & 0 \\
0 & 0 & 0 & 0 & 0 & N_{2}^{*} & 0 & B_{2}^{q^{*}}
\end{array}\right]
$$

where:

$$
\begin{aligned}
& F_{i}^{*}=\theta_{i} p_{i}^{*} \alpha_{i}^{j}\left(1-\omega_{j}\right), \quad G_{i}^{*}=-\theta_{i} p_{i}^{*} \beta_{i}^{j}\left(1-\nu_{j}\right), \\
& B_{i}^{p^{*}}=1+\theta_{i}\left(C_{i}^{p}-4 \alpha_{i}^{i} p_{i}^{*}+\beta_{i}^{i} q_{i}^{*}+\alpha_{i}^{j}\left(\omega_{j} p_{j}^{*}+\left(1-\omega_{j}\right) r_{j}^{*}\right)\right. \\
& \left.-\beta_{i}^{j}\left(\nu_{j} q_{j}^{*}+\left(1-\nu_{j}\right) s_{j}^{*}\right)\right), \quad B_{i}^{q^{*}}=1+\rho_{i}\left(-4 \vartheta_{i} q_{i}^{*}+\beta_{i}^{i} p_{i}^{*}-C_{i}^{q}\right), \\
& H_{i}^{*}=\theta_{i} p_{i}^{*} \alpha_{i}^{j} \omega_{j}, \quad L_{i}^{*}=\theta_{i} p_{i}^{*} \beta_{i}^{i}, \quad M_{i}^{*}=-\theta_{i} p_{i}^{*} \beta_{i}^{j} \nu_{j} \\
& \text { and } \quad N_{i}^{*}=\rho_{i} q_{i}^{*} \beta_{i}^{i} .
\end{aligned}
$$

such that $i, j \in\{1,2\}$ and $i \neq j$.

The characteristic polynomial $P_{8}(\lambda)$ of Jacobian matrix (28) has the form:

$P_{8}(\lambda)=\lambda^{8}+a_{7} \lambda^{7}+a_{6} \lambda^{6}+a_{5} \lambda^{5}+a_{4} \lambda^{4}+a_{3} \lambda^{3}+a_{2} \lambda^{2}+a_{1} \lambda+a_{0}$,

where:

$$
\begin{aligned}
a_{0} & =a_{1}=0 \\
a_{2} & =N_{2}^{*}\left(-N_{1}^{*} G_{1}^{*} G_{2}^{*}+F_{2}^{*} G_{1}^{*} B_{1}^{q^{*}}\right)+F_{1}^{*}\left(-F_{2}^{*} B_{2}^{q^{*}} B_{1}^{q^{*}}+N_{1}^{*} B_{2}^{q^{*}} G_{2}^{*}\right) \\
a_{3} & =N_{2}^{*} G_{1}^{*}\left(B_{1}^{q^{*}} H_{2}^{*}-F_{2}^{*}\right)-N_{1}^{*} N_{2}^{*}\left(G_{1}^{*} M_{2}^{*}+M_{1}^{*} G_{2}^{*}\right) \\
& +B_{2}^{q^{*}} H_{1}^{*}\left(N_{1}^{*} G_{2}^{*}-F_{2}^{*} B_{1}^{q^{*}}\right)+F_{1}^{*} N_{1}^{*}\left(B_{2}^{q^{*}} M_{2}^{*}-G_{2}^{*}\right) \\
& +F_{1}^{*} B_{2}^{q^{*}}\left(-B_{1}^{q^{*}} H_{2}^{*}+F_{2}^{*}\right)+F_{2}^{*} B_{1}^{q^{*}}\left(N_{2}^{*} M_{1}^{*}+F_{1}^{*}\right) \\
a_{4} & =B_{1}^{q^{*}} B_{2}^{q^{*}}\left(B_{2}^{p^{*}} B_{1}^{p^{*}}-H_{1}^{*} H_{2}^{*}\right)+N_{2}^{*} B_{1}^{q^{*}}\left(-L_{2}^{*} B_{1}^{p^{*}}+M_{1}^{*} H_{2}^{*}\right) \\
& +N_{1}^{*} N_{2}^{*}\left(L_{2}^{*} L_{1}^{*}-M_{1}^{*} M_{2}^{*}\right)+N_{1}^{*} B_{2}^{q^{*}}\left(-B_{2}^{p^{*}} L_{1}^{*}+H_{1}^{*} M_{2}^{*}\right)-F_{1}^{*} F_{2}^{*} \\
& -N_{2}^{*}\left(G_{1}^{*} H_{2}^{*}+F_{2}^{*} M_{1}^{*}\right)-N_{1}^{*}\left(H_{1}^{*} G_{2}^{*}+F_{1}^{*} M_{2}^{*}\right) \\
& +\left(F_{1}^{*} H_{2}^{*}+F_{2}^{*} H_{1}^{*}\right)\left(B_{1}^{q^{*}}+B_{2}^{q^{*}}\right) \\
a_{5} & =-H_{1}^{*}\left(F_{2}^{*}+N_{1}^{*} M_{2}^{*}\right)-H_{2}^{*}\left(F_{1}^{*}+N_{2}^{*} M_{1}^{*}\right)-B_{2}^{q^{*}} B_{1}^{p^{*}}\left(B_{1}^{q^{*}}+B_{2}^{p^{*}}\right) \\
& -B_{2}^{p^{*}} B_{1}^{q^{*}}\left(B_{2}^{q^{*}}+B_{1}^{p^{*}}\right)+H_{1}^{*} H_{2}^{*}\left(B_{1}^{q^{*}}+B_{2}^{q^{*}}\right) \\
& +N_{2}^{*} L_{2}^{*}\left(B_{1}^{p^{*}}+B_{1}^{q^{*}}\right)+N_{1}^{*} L_{1}^{*}\left(B_{2}^{q^{*}}+B_{2}^{p^{*}}\right) \\
a_{6} & =B_{2}^{q^{*}}\left(B_{1}^{p^{*}}+B_{1}^{q^{*}}\right)+B_{2}^{p^{*}}\left(B_{1}^{q^{*}}+B_{2}^{q^{*}}\right)+B_{1}^{p^{*}}\left(B_{1}^{q^{*}}+B_{2}^{p^{*}}\right) \\
& -H_{1}^{*} H_{2}^{*}-L_{1}^{*} N_{1}^{*}-L_{2}^{*} N_{2}^{*} \\
a_{7} & =-\left(B_{2}^{q^{*}}+B_{1}^{q^{*}}+B_{2}^{p^{*}}+B_{1}^{p^{*}}\right)
\end{aligned}
$$


The stability analysis can be reduced to that of determining conditions to ensure that all the roots of $P_{8}(\lambda)$ lie in the open unit disk $|\lambda|<1$. The stability conditions of Nash equilibrium are given by recalling Jury's conditions [12] which are the necessary and sufficient conditions for $\left|\lambda_{k}\right|<1, \quad k \in$ $\{1,2, \ldots, 8\}$. Consider the characteristic polynomial $P_{8}(\lambda)$ and let:

$$
\begin{aligned}
& b_{j}=a_{j}-a_{8-j} a_{0}, j=1 . .8 ; b_{0}=0 \\
& c_{j}=b_{j}-b_{8-j+1} \frac{b_{1}}{b_{8}}, j=2 . .8 ; c_{1}=c_{0}=0 \\
& d_{j}=c_{j}-c_{8-j+2} \frac{c_{2}}{c_{8}}, j=3 . .8 ; d_{2}=d_{1}=d_{0}=0 \\
& f_{j}=d_{j}-d_{8-j+3} \frac{d_{3}}{d_{8}}, j=4 . .8 ; f_{3}=f_{2}=f_{1}=f_{0}=0 \\
& g_{j}=f_{j}-f_{8-j+4} \frac{f_{4}}{f_{8}}, j=5 . .8 ; g_{4}=g_{3}=\ldots=g_{0}=0 \\
& h_{j}=g_{j}-g_{8-j+5} \frac{g_{5}}{g_{8}}, j=6 . .8 ; h_{5}=h_{4}=\ldots=h_{0}=0 \\
& k_{8}=h_{8}-h_{6} \frac{h_{6}}{h_{8}}, k_{7}=h_{7}-h_{7} \frac{h_{6}}{h_{8}}, k_{6}=k_{5}=\ldots=k_{0}=0 \\
& l_{8}=k_{8}-k_{7} \frac{k_{7}}{h_{8}}, l_{7}=l_{6}=l_{5}=\ldots=l_{0}=0
\end{aligned}
$$

a necessary and sufficient conditions for stability of Nash equilibrium $E_{*}$ are: $b_{8}>0, c_{8}>0, d_{8}>0, f_{8}>0, g_{8}>0$, $h_{8}>0, k_{8}>0$ and $l_{8}>0$.

\section{B. Numerical investigations}

Numerical experiments are simulated to show the stability and period doubling bifurcation route to chaos for system (27), in addition, we will show that the delay has the effect of increasing the stability domain. To do, it is suitable to take the parameters' values in Table 2:

\begin{tabular}{|c|c|c|c|c|}
\hline$\alpha_{1}^{\perp}=\alpha_{2}^{2}$ & $\alpha_{2}^{\perp}=\alpha_{1}^{2}$ & $\beta_{1}^{\perp}=\beta_{2}^{2}$ & $\beta_{2}^{\perp}=\beta_{1}^{2}$ & $D_{0}^{\perp}=D_{0}^{2}$ \\
\hline 0.7 & 0.3 & 0.7 & 0.3 & 20 \\
\hline \hline$\nu_{1}=\nu_{2}$ & $\omega_{1}=\omega_{2}$ & $\vartheta_{1}=\vartheta_{2}$ & $\theta_{i}$ & $\rho_{i}$ \\
\hline 0.5 & 0.5 & 1 & $0<\theta_{i}<0.1$ & $0<\rho_{i}<0.1$ \\
\hline
\end{tabular}

TABLE II

PARAMETERS' VALUES USED FOR NUMERICAL SIMULATIONS.

In order to study the influence of delay $\omega_{i}$ on the stability of both price and QoS equilibrium, we plot respectively in Figures 8 and 9 the bifurcation of price and QoS decided by SP-1 w.r.t $\theta_{1}$ in two cases: no-delay case $\left(\omega_{1}=\omega_{2}=1\right)$ and delay case $\left(\omega_{1}=\omega_{2}=0.5\right)$. On the one hand we note that the bifurcation diagram of $p_{1}$ and $q_{1}$ in the non-delay case $\omega_{1}=\omega_{2}=1$ converges to Nash equilibrium as $\theta_{1}<0.032$, by cons, when $\theta_{1}>0.032$ the Nash equilibrium of price and QoS becomes unstable, period doubling bifurcation appear and after chaotic behaviors occur for both price and QoS diagrams. Also the bifurcation diagrams of $p_{1}$ and $q_{1}$ w.r.t $\theta_{1}$ in delay case are plotted, in this case, the Nash equilibrium is converges as $\theta_{1}<$ 0.038 , when $\theta_{1}>0.038$ the Nash equilibrium point becomes unstable. On the other hand, it is observed that that period doubling bifurcation are delayed in delay case, so, we deduce that delay $\omega_{i}$ increases the stability of the Nash equilibrium.

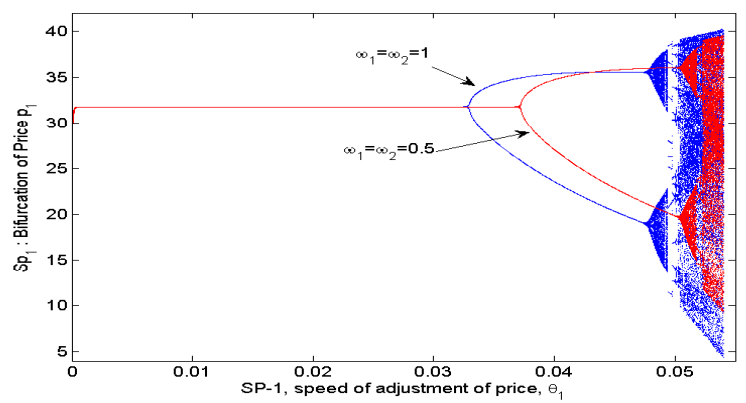

Fig. 8. Bifurcation diagrams of $p_{1}$ with respect to $\theta_{1}$ with (red) and without (blue) delay, where $\theta_{2}=0.03, \rho_{i}=0.05$ and $\nu_{i}=0.5$.

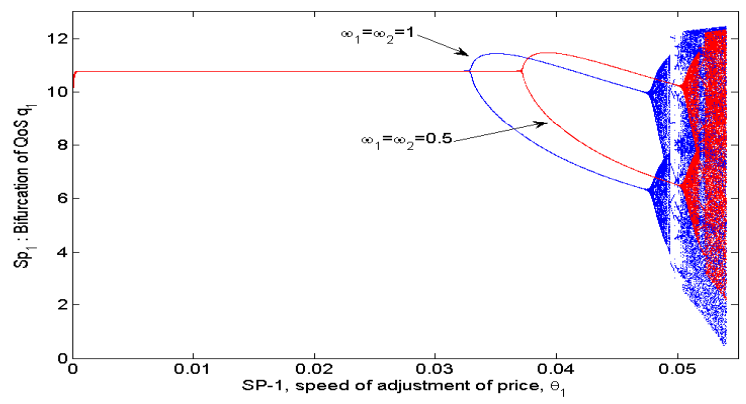

Fig. 9. Bifurcation diagrams of $q_{1}$ with respect to $\theta_{1}$ with (red) and without (blue) delay, where $\theta_{2}=0.03, \rho_{i}=0.05$ and $\nu_{i}=0.5$.

Now we study the influence of delay $\nu_{i}$ on the stability of both price and QoS equilibrium, we plot respectively in Figures 10 and 11 the bifurcation of price and QoS decided by SP-1 w.r.t $\rho_{1}$ in two cases: no-delay case $\left(\nu_{1}=\nu_{2}=1\right)$ and delay case $\left(\nu_{1}=\nu_{2}=0.5\right)$. Bifurcation diagram of $p_{1}$ and $q_{1}$ in the non-delay case $\nu_{1}=\nu_{2}=1$ converges to Nash equilibrium as $\rho_{1}<0.030$, contrariwise, when $\theta_{1}>0.030$ the Nash equilibrium of price and QoS becomes unstable, period doubling bifurcation appear and after chaotic behaviors occur for both price and QoS diagrams. Comparing the bifurcation diagrams of each Figure (Figures 10 and 11) we find that delay $\nu_{i}$ affects the stability of Nash equilibrium point, and that SPs using delayed bounded rationality have a higher chance of reaching a Nash equilibrium point.

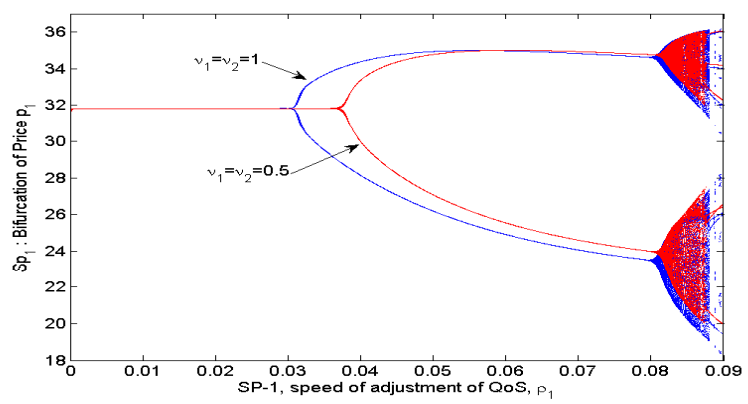

Fig. 10. Bifurcation diagrams of $p_{1}$ with respect to $\rho_{1}$ with (red) and without (blue) delay, where $\rho_{2}=0.04, \theta_{i}=0.04$ and $\nu_{i}=0.5$. 


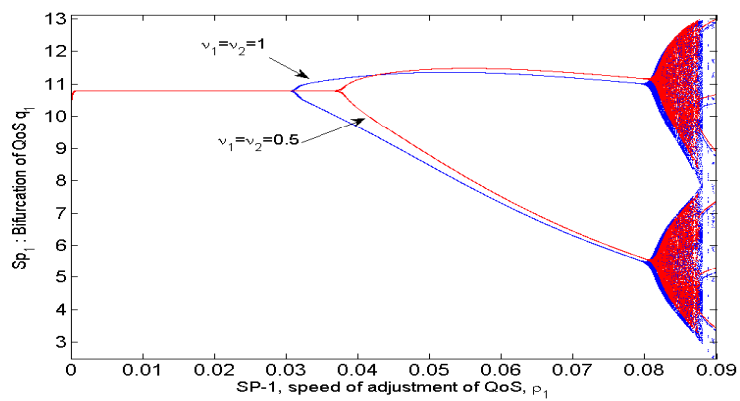

Fig. 11. Bifurcation diagrams of $q_{1}$ with respect to $\rho_{1}$ with (red) and without (blue) delay, where $\rho_{2}=0.04, \theta_{i}=0.04$ and $\nu_{i}=0.5$.

In Figures 12 and 13, we present simultaneously the price and QoS of SP-1 at equilibrium w.r.t $\theta_{1}$ and $\rho_{1}$.

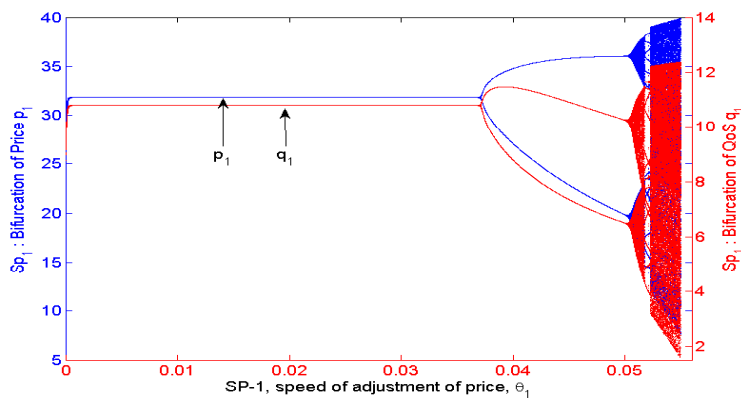

Fig. 12. Bifurcation diagrams of $p_{1}$ (blue) and $q_{1}$ (red) with respect to $\theta_{1}$, where $\theta_{2}=0.03, \rho_{i}=0.05$ and $\omega_{i}=\nu_{i}=0.5$.

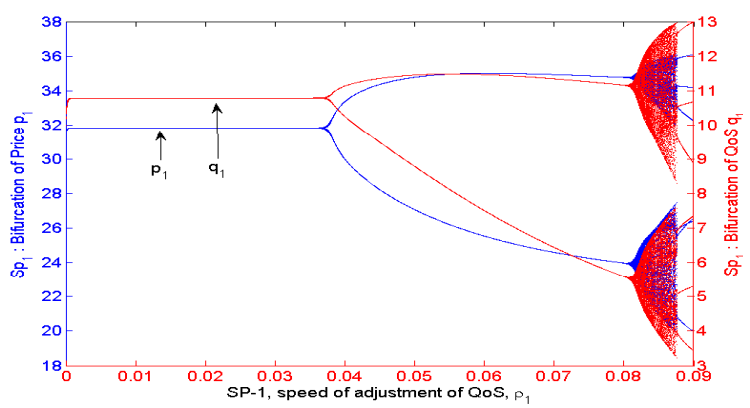

Fig. 13. Bifurcation diagrams of $p_{1}$ (blue) and $q_{1}$ (red) with respect to $\rho_{1}$, where $\rho_{2}=0.03, \theta_{i}=0.04$ and $\omega_{i}=\nu_{i}=0.5$.

\section{Conclusion}

We studied the problem of price and QoS game for two SPs assuming multi-level rationality under two schemes : Symmetric scenario where both SPs are rational, and asymmetric scenario where one of them is rational and the second SP has limited knowledge. We first provided many conditions for stability of Nash equilibria. Second, we showed that the delay enhances the stability of the equilibrium for both schemes. Furthermore, we studied a duopoly game with bounded rationality while considering both price and QoS as strategic parameters.
Stability conditions of Nash equilibria and the impact of the memory size are also discussed. Results found in this work can be further extended to general network considerations, in particular under non-neutrality perspective or non-linear demand.

\section{REFERENCES}

[1] M. Baslam, L. Echabbi, R. El Azouzi and E. Sabir, "Joint Price and QoS Market Share Game with Adversarial Service Providers and Migrating Customers", Proc. of GameNets, China, 2011.

[2] R. El-Azouzi, E. Altman and L. Wynter, "Telecommunications Network Equilibrium with Price and Quality-of-Service Characteristics", Proc. of ITC, Berlin, Sept 2003.

[3] J. von Neumann and O. Morgenstern, "Theory of Games and Economic Behavior", Princeton University Press, 1944.

[4] A. Orda, R. Rom and N. Shimkin, "Competitive routing in multi-user environments", IEEE/ACMTrans. on Netw., 510-521, 1993.

[5] A. A. Elsadany, "Dynamics of a delayed duopoly game with bounded rationality", in Mathematical and Computer Modelling, Vol. 52, Nr. 9-10 (2010) , p. 1479-1489.

[6] E. Ahmed, H.N. Agiza, S.Z Hassan, "On modification of Puu s dynamical duopoly", Chaos, Solitons and Fractals 11 (7) (2000) 10251728.

[7] M.T. Yassen, H.N. Agiza, "Analysis of a duopoly game with delayed bounded rationality", In Applied Mathematics and Computation, Vol. 138, N. 2-3, pp. 387-402, 2003.

[8] R.L. Devaney, "An Introduction to chaotic dynamical systems". Reading, MA: Addison-Wesley; 1989.

[9] G.I. Bischi, A. Naimzada, "Global analysis of a dynamic duopoly game with bounded rationality", in: Advanced in Dynamics Games and Application, vol. 5, Birkhauser, Basel, 1999, Chapter 20.

[10] R. Gibbons, "A Primer in Game Theory", Simon and Schuster, New York, 1992.

[11] A. Stiefenhofer, "Stability and predictability of dynamical economic systems with memory", discussion paper 331, Department of Economics, Bielefeled University, 1997.

[12] X. Li, C. Mou, W. Niu, and D. Wang, "Stability Analysis for Discrete Biological Models Using Algebraic Methods", in: Mathematics in Computer Science, pp.247-262, 2011.

[13] M. Baslam, R. El-Azouzi, E. Sabir and E. H. Bouyakhf, "Technical Report : New Insights from a Bounded Rationality Analysis for Strategic Price-QoS War". http://lia.univ-avignon.fr/fileadmin/documents/ Users/Intranet/chercheurs/sabir/BoundedRationality.pdf 